\title{
Fabry-Pérot filter cavities for wide-spaced frequency combs with large spectral bandwidth
}

\author{
T. Steinmetz • T. Wilken • C. Araujo-Hauck • \\ R. Holzwarth · T.W. Hänsch • T. Udem
}

Received: 22 December 2008 / Published online: 13 February 2009

(C) The Author(s) 2009. This article is published with open access at Springerlink.com

\begin{abstract}
We use low-finesse Fabry-Pérot cavities in series to generate frequency combs with a large mode spacing in a way that allows its application to a large optical bandwidth. The attenuation of laser modes closest to the pass bands of the cavity exceeds $70 \mathrm{~dB}$ for a filter ratio of $m=20$ relative to the resonant modes centered within the pass bands. We also identify the best cavity geometry to suppress spurious transmission of higher order transversal modes. Such a thinned out frequency comb can be used to calibrate traditional spectrographs for precision astronomy. In the time domain mode filtering generates a pulse train with a multiplied repetition rate. High-fidelity filtering, as described here, implies small variations of the pulse energies.
\end{abstract}

PACS 42.60.Da · 42.60.Fc · 42.62.Eh · 95.10.Jk

\section{Introduction}

The extremely regular spacing of longitudinal cavity modes of a passively mode-locked laser has proven to be a powerful tool for optical frequency metrology. The mode spacing

T. Steinmetz $(\bowtie) \cdot$ T. Wilken · R. Holzwarth · T.W. Hänsch ·

T. Udem

Max-Planck-Institut für Quantenoptik, Hans Kopfermann Str. 1,

85748 Garching, Germany

e-mail: tilo.steinmetz@mpq.mpg.de

Fax: +49-89-32905200

T. Steinmetz $\cdot$ R. Holzwarth

Menlo Systems GmbH, Am Klopferspitz 19, 82152 Martinsried, Germany

C. Araujo-Hauck

European Southern Observatory, Karl-Schwarzschild-Str. 3, 85748 Garching, Germany of such a comb is given by the pulse repetition rate that depends on the type of the laser and is typically on the order of $100 \mathrm{MHz}$. For sufficiently broad spectra each mode can be phase coherently stabilized relative to an atomic clock thereby transferring the clocks accuracy in a single step to the optical domain. This provides the means to perform absolute optical frequency measurement with the accuracy of an atomic clock [1].

Besides applications in laser spectroscopy, fundamental physics, and optical atomic clocks, frequency combs may also be used to calibrate traditional spectrographs. In this case the comb acts as a transfer device that allows to map a reference frequency to light sources whose frequency cannot be measured easily. A prominent example is astronomy where Doppler methods are used to determine cosmic velocities such as the Hubble flow of galaxies. Another astronomical application is to detect small extrasolar planets through the quiver motion that they impose on the stars that they orbit $[2,3]$.

Extremely stable astronomical spectrographs have been developed that can maintain their calibration with up to 4 parts in $10^{9}$ over months [4]. To achieve absolute frequency calibration iodine absorption cells or Thorium-Argon lamps have been used so far. They suffer from their limited reproducibility, aging, line blending, and from irregular line spacings and intensities. Using a frequency comb for calibration non of these effects should be an issue. The frequency comb may also be adjusted for the optimum mode spacing which is just above the resolution of the instrument [5]. In this way, small corrections to the spectrometers calibration curve may be determined that are due to a variety reasons, like imperfect optics, pixel position, and sensitivity variations to name a few. In addition, reproducibility over many years is particularly important if changes in the Hubble flow rate, i.e., the acceleration of the universe or long-orbit extrasolar planets 


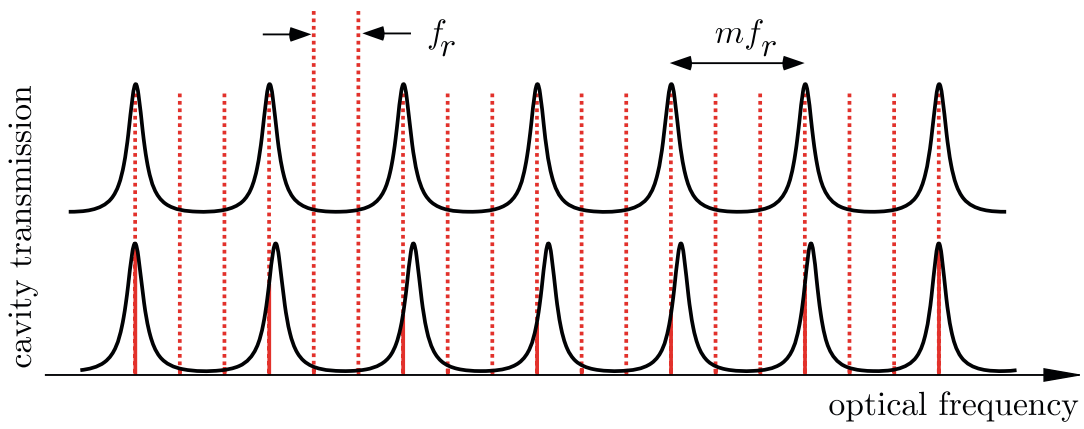

Fig. 1 Top: Matching up the modes of an (always) perfectly regular frequency comb (dotted) with the modes of a perfect filter cavity (solid) with filter ratio of $m=4$ and mirror reflectivity of $R=95 \%$. Bottom: Cavity dispersion that is nonlinear in frequency (GVD) destroys the

are investigated. The frequency comb can readily achieve this by referencing to an atomic clock so that results are archived and may be compared with results obtained by future instruments.

The relative line spacings within the redshifted fine structure multiplets observed in absorption of gas clouds that are back-illuminated by continuous light from a quasar provides a sensitive probe for a possible slow variation of the fine-structure constant [6]. Recent data obtained for that purpose has an uncertainty of a few parts in $10^{7}$. So far only large extrasolar planets in close orbit ("hot Jupiters") have been detected because of the limited sensitivity of $\sim 30 \mathrm{~m} / \mathrm{s}$ (one part in $10^{7}$ ). The resolution required to detect an Earth-like system which recoils with $v=9 \mathrm{~cm} / \mathrm{s}$, is $v / c=3 \times 10^{-10}$. The direct detection of the acceleration of the universe within 20 years of observation time requires even a resolution of $v / c=3 \times 10^{-11}$ [2].

Whereas a frequency comb can easily meet the accuracy requirements for the above applications, say with a simple GPS disciplined rubidium clock available for few $\mathrm{k} \$$, attaining the required mode spacing, i.e., repetition rate, is more challenging. For this it should be noted that astronomical spectrographs also need to be optimized for signal to noise ratio to make faint objects detectable. Therefore the useful resolution is restricted in order not to distribute the available number of photons among too many detectors or CCD pixels. In practical terms this means that the mode spacing for the present and next generation instruments should be on the order of $10 \mathrm{GHz}$ or above. Titanium sapphire lasers with such a high repetition rate that also provide the spectral width to be useful for precision astronomy are indeed feasible [7]. Even though it may be possible to further increase this repetition rate, Ti:sapphire lasers so far require continuous attention that adds significant complexity when spectra have to be averaged for long observation times. An alternative would be to operate mode-locked lasers not with a single intracavity pulse but with an intracavity train of pulses regular mode spacing leading to misalignment of the filter for some of the modes (exaggerated). In addition, when combined with residual frequency noise of the frequency comb, this can skew the spectrum of the misaligned modes

with constant temporal spacing [8] or to use an intracavity Fabry-Pérot filter to generate multiply pulses [9]. In these cases though the limited available average power of most lasers is distributed among more pulses per second so that the necessary peak power for spectral broadening is difficult to achieve. So far spectral broadening, for example in non linear fibers, seems to be necessary as the lasers themselves cannot supply the required optical bandwidth. A broad optical spectrum not only calibrates the spectrograph across the complete operating range, which is typically an octave worth of optical frequencies, but also allows to stabilize the frequency comb to an atomic clock [1]. So we are left with conflicting requirements: large repetition rate for large mode spacing and low repetition rate for large peak intensity.

For previously noted reasons we have focused our research on mode-locked fiber lasers that are spectrally filtered by external Fabry-Pérot filter cavities $[10,11]$ to thin out the modes from the frequency comb as shown in the upper part of Fig. 1. Besides the capability of fiber lasers to operate without human attention for months, the filtered pulses with significantly reduced pulse energy may be readily reamplified with a fiber amplifier. This is essential if spectral broadening of the filtered pulse train is required. On the other hand, one might want to broaden the spectrum before the Fabry-Pérot filter where the peak intensity is still high. However, as explained in the next paragraph, this will result in severe requirements on the dispersion properties of that filter cavity.

A good suppression of the attenuated modes is essential for calibration purposes because eventually individual lines are deconvoluted with the known point spread function of the spectrograph for the ultimate resolution. Insufficient suppression of side modes that lie within the resolution of the spectrograph will result in systematic shifts of the calibration curve. Therefore a high-finesse Fabry-Pérot filter cavity seems to be required. On the other hand, statistical calibrating uncertainties are reduced when a large optical bandwidth 


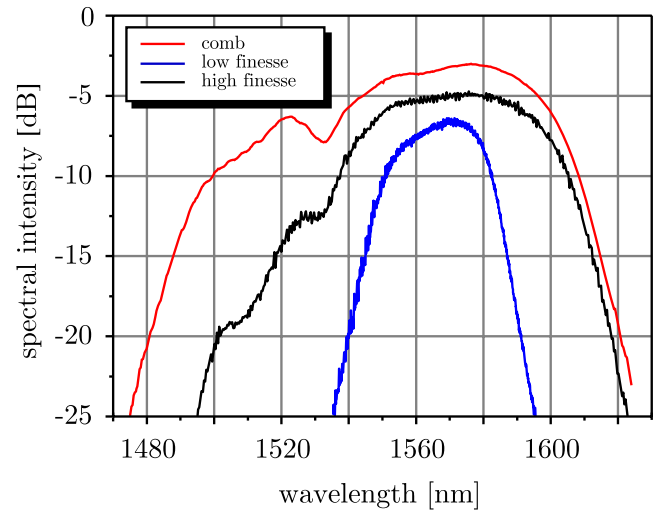

Fig. 2 Optical spectrum of an erbium-doped fiber frequency comb (upper, red) and the corresponding spectrum filtered by a low-finesse (middle, black) and a high-finesse cavity (lower, blue) of $F \sim 400$ and $F \sim 2400$, respectively. Curves have been offset vertically for clarity

is available as mentioned earlier. Unfortunately, imperfectly compensated group velocity dispersion (GVD) of the mirror coatings generates small irregularities of the filter mode spacing which are detrimental for a filter cavity with a large finesse (see upper part of Fig. 1). So far this has prevented the filtering technique from being effective over a wide optical bandwidth (see Fig. 2). In addition, non perfect alignment of the filter modes with the modes of the frequency comb leads to a systematic shift of the intensity weighted center of the transmitted spectrum relative to the stabilized mode frequency when frequency noise is present. This can cause systematic uncertainties of the obtained calibration of the spectrograph as pointed out in Ref. [12]. Again we find conflicting requirements: high finesse for good side-mode suppression and low finesse for insensitivity to group velocity mismatch. We believe that we can also offer a solution to this problem by combining several low-finesse filter cavities in series.

\section{Combining filter cavities in series}

Rather than using several cavities after spectral broadening that filter spectral slices in parallel as proposed in Ref. [12], we are using the cavities in series before spectrally broadening. A single two-mirror Fabry-Pérot filter cavity without dispersion made from mirrors of reflectivity $R$ that are separated by a distance $L$ has a spectral transmission function of

$$
T(f, R, L)=\frac{(1-R)^{2}}{(1-R)^{2}+4 R \sin ^{2}(2 \pi f L / c)}
$$

as shown in the upper part of Fig. 1. Dispersion can be taken into account by replacing $L$ with the optical path length. Without it, it implies a regular grid of transmissions, each

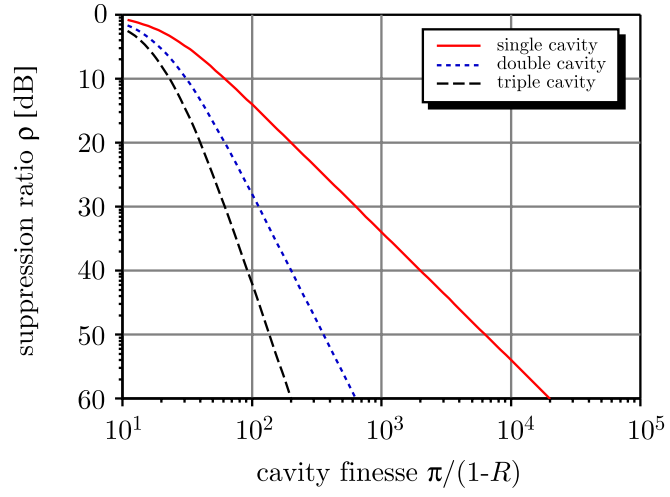

Fig. 3 Side-mode suppression ratio as defined in the text for single (red), double (blue, dotted), and triple (black, dashed) filter cavities with filter ratio $m=40$

$\Delta f=\operatorname{FSR}(1-R) / \pi$ wide, with the free spectral range $\mathrm{FSR}=c / 2 L$. For our purpose the filter mode spacing is set to an integer multiple $m$ of the laser comb spacing $f_{r}$ by adjusting its length such that $m f_{r}=c / 2 L$. The filter cavity then transmits exactly every $m$-th mode while the unwanted modes in between are largely suppressed. We define the side-mode suppression ratio $\rho$ by residual power transmission of the mode closest to but not on the cavity transmission by $\rho=T\left(f_{r}, R, L\right)$. An upper limit of the observed spectral shift due to the presence of side modes can be obtained by assuming only one of them to be present. The center of mass of the transmitted pair of frequency comb components is calculated as the intensity weighted $(1$ and $\rho)$ average with frequency difference $f_{r}$ to

$\Delta f=\frac{0+\rho f_{r}}{1+\rho} \approx \rho f_{r}$.

To ensure that the above requirements of $\Delta f / f \sim 3 \times 10^{-11}$ are fulfilled, with some safety margin for say the visible $f \approx 500 \mathrm{THz}$, we want to achieve $\sim \rho=50 \mathrm{~dB}$. In order to convert from a $100 \mathrm{MHz}$ to a $10 \mathrm{GHz}$ frequency comb with a single cavity $(m=100)$ a finesse of $F=$ $\pi /(1-R)=1600(R=99.8 \%)$ would be required. Such a finesse is readily available for single mode lasers, but would be difficult to obtain for broad-band operation, not to mention the noise associated spectral skewing [12]. Concatenating several cavities in series the transmission function $T(f, R, L)$ and the side-mode suppression multiplies while the requirement for the mirror reflectivities are alleviated.

In Fig. 3 we show the side-mode suppression for single, double, and triple cavities with a filter ratio of $m=40$ as a function of cavity finesse. Our fiber based frequency comb [13] operates with a repetition rate of $250 \mathrm{MHz}$ so that $m=40(L=1.5 \mathrm{~cm})$ is an appropriate choice. In this case a double cavity arrangement with $F=400(R=99.2 \%)$ achieves the required $50 \mathrm{~dB}$ side-mode suppression. With 
Fig. 4 Several possibilities to combine two filter cavities to thin out the modes of a frequency comb (FC). Double pass through a single cavity by using orthogonal

polarizations (a). Cascaded cavities; two linear cavities separated by an optical isolator (b), two ring resonators $(\mathbf{c})$

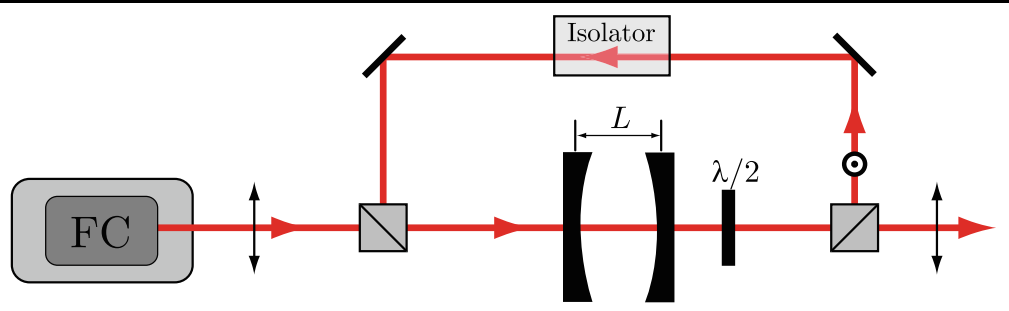

a

double pass

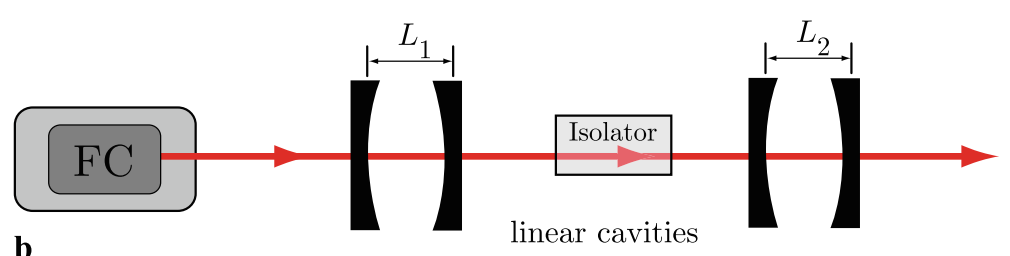

b

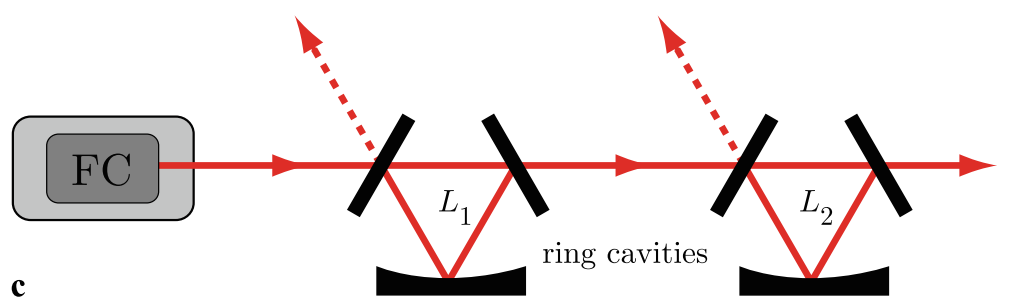

Fig. 5 Scheme of the filtering setup: A frequency comb (FC) generated by a mode-locked fiber laser is filtered by means of two low-finesse Fabry-Pérot cavities in series to obtain a large mode spacing and to maintain a high optical bandwidth. After the first filtering stage the signal is amplified by a fiber amplifier (EDFA). The reflected light on each cavity is detected with a split photodiode in order to generate an error signal for cavity locking

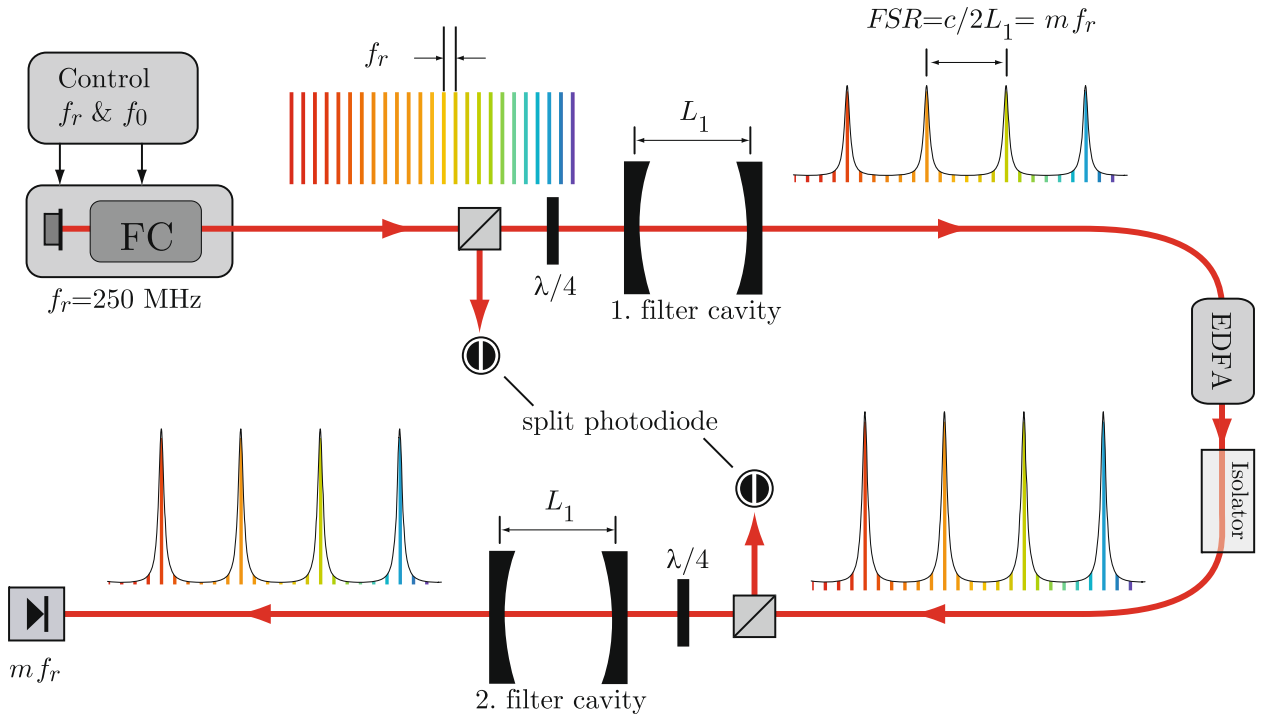

a triple mirror arrangement the requirements would already be down to $F=100$ ( $R=96.9 \%$ ) for the same condition. In this case metallic mirrors that are broad band with virtually no dispersion can be used virtually eliminating the bandwidth limiting dispersion.

When placing more than one filter cavity in series one has to either prevent the formation of additional cavities in between them or make use of them by adjusting their free spectral ranges. There are several possibilities to accomplish this. One might even use a single cavity in double pass by rotating the polarization on the second transmission. This could be done collinearly or with counter propagating beams. Another possibility to avoid spurious cavities is to use ring cavities or optical isolators. Some of these possibilities are shown in Fig. 4.

To demonstrate this scheme we have used two filter cavities so far constructed from dielectric mirrors with a finesse around $400(R=99.2 \%)$ and a FSR of $5 \mathrm{GHz}$, and with an identical filter ratio of $m=20$ as shown in Fig. 5. The sidemode suppression of the singly filtered frequency comb is calculated to be $32 \mathrm{~dB}$ and for the doubly filtered comb to be $64 \mathrm{~dB}$, in good agreement with the experiment. Filtering 

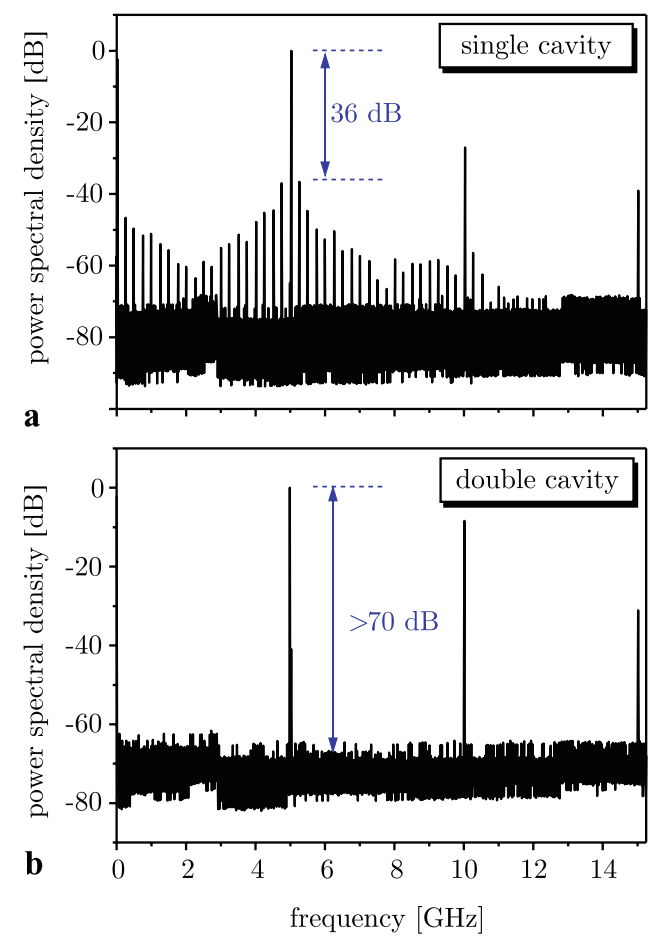

Fig. 6 A frequency comb with $250 \mathrm{MHz}$ repetition rate is filtered with one (a) and two (b) Fabry-Pérot cavities. Both cavities have a free spectral range of $5 \mathrm{GHz}$ and a finesse of $\sim 400$. The suppression ratio is determined by recording the RF spectrum of the transmitted pulse train and is found to exceed $70 \mathrm{~dB}$ for double filtering. The decrease in amplitude for the transmitted modes for higher frequencies is due to the limited bandwidth of the detector

the comb to the same level in a single step, a cavity with finesse of $16000(R=99.98 \%)$ would be needed. To identify the filtered mode number we lock a continuous wave $(\mathrm{CW})$ laser to a comb line while its frequency is measured by a wavemeter that is sufficiently accurate (better than $f_{r}$ ) [2].

In Fig. 6 the measured transmitted rf-spectrum of a filtered frequency comb is shown for single and double filtering. The proper measurement of the side-mode suppression would involve generating beat notes of the filtered comb with a continuous wave laser. The relative strengths of the beat notes as observed on a radio frequency spectrum analyzer then reflects the mode suppression. A simpler method, that does not require a continuous wave laser, is the direct detection of the filtered pulse train. In this case the strongest frequency component should be $m f_{r}$ whereas the closest side band shows up with a frequency $f_{r}$. In the frequency domain these components arise as intermode beatings. It turns out that there are twice as many combinations that lead to a frequency of $f_{r}$ than there are that lead to $m f_{r}$. Therefore the side-mode suppression measured in this way is $3 \mathrm{~dB}$ larger than $\rho$. Similar arguments can be applied for the strengths of the higher order side bands.

For stabilizing the cavity length we use the so-called tilt locking method [14]. This technique uses the spatial modes interference between the carrier field $\left(\mathrm{TEM}_{00}\right)$ and a directly reflected, nonresonant higher order mode $\left(\mathrm{TEM}_{01}\right)$ as a phase reference to derive an error signal for the feedback phase. To separate the transverse electric-field amplitudes the reflected light from the cavity is detected on a two-element split photodiode. The advantage of this locking scheme is that no modulation is required but other schemes may work as well.

\section{Optimum mirror radius of curvature}

The suppression factor $\rho$ was calculated in Sect. 2 with the assumption of perfect spatial mode coupling of the frequency comb to the $\mathrm{TEM}_{00}$ mode of the filter cavities. However, for non perfect mode matching higher order transverse modes of the filter cavities may get close to or even coincide with modes of the frequency comb that we want to suppress. This not only spoils the filtered frequency comb, but will also make the side-mode suppression coupling and alignment dependent so that it might change over time.

There is, however, a simple way around that problem by selecting appropriate values for the radius of curvature for the filter cavity mirrors. With a suitable choice the transversal modes can be arranged such that they are located in between the modes of the frequency comb. The resonance frequencies of a cavity made from mirrors with radius of curvature $r_{1}$ and $r_{2}$ are given by

$v_{q, i, j}=\frac{c}{2 L}\left(q+\frac{1+i+j}{\pi} \arccos \left(\sqrt{g_{1} g_{2}}\right)\right)$

with the longitudinal and transversal mode numbers $q, i$, and $j$ respectively and $g_{i}=1-L / r_{i}$ [15]. Assuming the length $L$ to be fixed to the desired filter ratio $L=m c / 2 f_{r}$, the radii may be chosen within the stability limit of the cavity.

In Fig. 7 we show some examples of good and bad arrangements by assuming exponentially reducing spatial overlap with transversal mode numbers $i+j$. It can be seen that with a smart choice of $r_{i}$ this problem can be virtually eliminated, for reasonable mode matching to the $\mathrm{TEM}_{00}$.

In conclusion, we have demonstrated a new technique for mode filtering of frequency combs that addresses the issues of broad-band operation and high extinction ratio of unwanted side modes. With just one mode filter cavity it is difficult to achieve the high finesse that is needed for a sidemode suppression on the order of $50 \mathrm{~dB}$ and flat GVD over hundreds of nanometers of spectral bandwidth. The solution to this dilemma is the use of multiple lower finesse cavities in series. With such an arrangement we have demonstrated very high side-mode extinction exceeding $70 \mathrm{~dB}$ for a filter ratio of $m=20$ with just two cavities operated at the same free spectral range. Metallic mirrors with virtually no GVD may be used in such an arrangement. In addition we discuss 


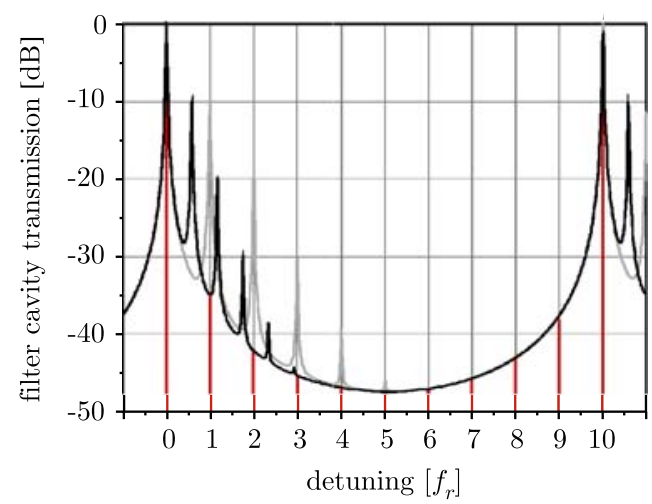

Fig. 7 Left: Frequency comb filtered by a $m=10$ filter ratio with $R=$ $99.2 \%$ and a suitable choice of mirror curvatures $r_{1}=\infty, r_{2}=30 L$. The transversal modes $i+j=1,2,3,4,5$ are assumed to spatially overlap with the beam from the comb generator by $-10,-20,-30$, -40 , and $-50 \mathrm{~dB}$, respectively, relative to the TEM $\mathrm{T}_{00}$. The unwanted modes from the frequency comb (red vertical lines) are suppressed by at least $35 \mathrm{~dB}$ (black curve). However, for an unfavorable choice,

the possibility to adjust the higher order modes of the filter cavities in order to avoid coincidences with the attenuated modes from the frequency comb.

Acknowledgements Partial support by the DFG cluster of excellence Munich Centre for Advanced Photonics (MAP) is acknowledged. The authors would like to thank Luca Pasquini and Antonio Manescau from the European Southern Observatory, Garching Germany and Michael T. Murphy from the Centre for Astrophysics and Supercomputing, Swinburne University, Australia for very helpful discussions about astrophysics, cosmology, and instrumentation.

Open Access This article is distributed under the terms of the Creative Commons Attribution Noncommercial License which permits any noncommercial use, distribution, and reproduction in any medium, provided the original author(s) and source are credited.

\section{References}

1. Th. Udem, R. Holzwarth, T.W. Hänsch, Nature 416, 233 (2002)

2. T. Steinmetz, T. Wilken, C. Araujo-Hauck, R. Holzwarth, T.W. Hänsch, L. Pasquini, A. Manescau, S. D’Odorico, M.T. Murphy, T. Kentischer, W. Schmidt, T. Udem, Science 321, 1335 (2008)

3. C.H. Li, A.J. Benedick, P. Fendel, A.G. Glenday, F.X. Kärtner, D.F. Phillips, D. Sasselov, A. Szentgyorgyi, R.L. Walsworth, Nature 452, 610 (2008)

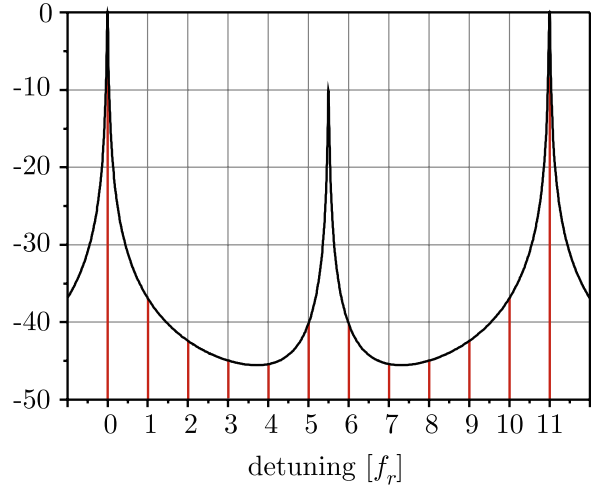

$r_{1}=\infty, r_{2}=10.47 L$, the higher order transversal modes of the filter cavity all coincide with the unwanted modes of the frequency comb (grey curve). Right: a good choice could also be a confocal cavity with $r_{1}=r_{2}=L\left(g_{1}=g_{2}=0\right)$ if the filter ratio $m$ is odd and the cavity finesse sufficiently large. In this example $(R=99.2 \%)$ the side-mode suppression is at least $40 \mathrm{~dB}$

4. C. Lovis, F. Pepe, F. Bouchy, G. Lo Curto, M. Mayor, L. Pasquini, D. Queloz, G. Rupprecht, S. Udry, S. Zucker, Proc. SPIE 6269, 62690P-1 (2006)

5. M.T. Murphy, Th. Udem, R. Holzwarth, A. Sizmann, L. Pasquini, C. Araujo-Hauck, H. Dekker, S. D’Odorico, M. Fischer, T.W. Hänsch, A. Manescau, Mon. Not. R. Astron. Soc. 380, 839 (2007)

6. J.K. Webb, M.T. Murphy, V.V. Flambaum, V.A. Dzuba, J.D. Barrow, C.W. Churchill, J.X. Prochaska, A.M. Wolfe, Phys. Rev. Lett. 87, 091301 (2001)

7. A. Bartels, D. Heinecke, S.A. Diddams, Opt. Lett. 33, 1905 (2008)

8. S. Zhou, D.G. Ouzounov, F.W. Wise, Opt. Lett. 31, 1041 (2006)

9. T.M. Liu, F.X. Kärtner, J.G. Fujimoto, C.K. Sun, Opt. Lett. 30, 439 (2005)

10. T. Sizer, IEEE J. Quantum Electron. 25, 97 (1989)

11. Th. Udem, J. Reichert, R. Holzwarth, T.W. Hänsch, Phys. Rev. Lett. 82, 3568 (1999)

12. D.A. Braje, M.S. Kirchner, S. Osterman, T. Fortier, S.A. Diddams, Eur. Phys. J. D 48, 57 (2008)

13. T. Wilken, T.W. Hänsch, R. Holzwarth, P. Adel, M. Mei, in Conference on Lasers and Electro-Optics (CLEO) 2007, Paper: CMR3

14. D.A. Shaddock, M.B. Gray, D.E. McClelland, Opt. Lett. 24, 1499 (1999)

15. E.A. Siegman, Lasers (University Science Books, Herndon, 1986) 